\title{
Status of the LHCf experiment
}

\author{
Hiroaki Menjo ${ }^{a *}$, Oscar Adriani ${ }^{b c}$, Eugenio Berti $^{b c}$, Lorenzo Bonechi $^{b}$, \\ Massimo Bongi ${ }^{b c}$, Guido Castellini ${ }^{d}$, Raffaello D'Alessandro ${ }^{a b}$, \\ Maurice Haguenauer ${ }^{e}$, Yoshitaka Itow ${ }^{f g}$, Katsuaki Kasahara ${ }^{h}$, Kimiaki Masuda $^{f}$, \\ Yutaka Matsubara $^{f}$, Yasushi Muraki $^{f}$, Ken Oohashi $^{f}$ Pauro Papini $^{b}$, \\ Sergio Ricciarini ${ }^{b d}$, Takashi Sako ${ }^{f g}{ }$ Nobuyuki Sakurai $^{i}$, Kenta Sato $^{f}$, Yuki Shimizu $^{j}$, \\ Maiko Shinoda $^{f}$, Takuya Suzuki $^{h}$, Tadashi Tamura $^{j}$, Alessio Tiberio ${ }^{b}$, Shoji Torii $^{h}$, \\ Alessia Tricomi $^{k l}$, Bill Turner ${ }^{n}$, Mana Ueno ${ }^{f}$, Kenji Yoshida ${ }^{n}$, and Qudong Zhou ${ }^{f}$ \\ ${ }^{a}$ Graduate School of Science, Nagoya University, Nagoya, Japan \\ ${ }^{b}$ INFN Section of Florence, Florence, Italy \\ ${ }^{c}$ University of Florence, Florence, Italy \\ ${ }^{d}$ IFAC-CNR, Florence, Italy \\ ${ }^{e}$ Ecole-Polytechnique, Palaiseau, France \\ ${ }^{f}$ Institute for Space-Earth Environmental Research, Nagoya University, Nagoya, Japan \\ ${ }^{g}$ Kobayashi-Maskawa Institute for the Origin of Particles and the Universe, Nagoya University, \\ Nagoya, Japan \\ ${ }^{h}$ RISE, Waseda University, Shinjuku, Tokyo, Japan \\ ${ }^{i}$ Tokushima University, Tokushima, Japan \\ ${ }^{j}$ Kanagawa University, Kanagawa, Japan \\ ${ }^{k}$ INFN Section of Catania, Italy \\ ${ }^{l}$ University of Catania, Catania, Italy \\ ${ }^{m}$ LBNL, Berkeley, California, USA \\ ${ }^{n}$ Faculty of System Engineering, Shibaura Institute of Technology, Japan \\ E-mail:menjo@isee.nagoya-u.ac.jp
}

The LHCf experiment is an LHC experiment dedicated to measure the production spectra of forward neutral particles, photons, $\pi^{0}$ 's, and neutrons. The aim of the LHCf is to provide critical data to test and tune hadronic interaction models which are used in MC simulations for cosmic-ray air shower developments. The LHCf had an operation in 2015 with pp collisions at $\sqrt{s}=13 \mathrm{TeV}$, which corresponds to the collision energy of $0.9 \times 10^{17} \mathrm{eV}$ in the laboratory frame. The resent results of the LHCf, the inclusive energy spectra for forward photons and neutrons obtained with pp collisions at $\sqrt{s}=13 \mathrm{TeV}$, are presented. In addition, future prospects of LHCf analyses and activities are reviewed.

35th International Cosmic Ray Conference - ICRC2017

10-20 July, 2017

Bexco, Busan, Korea

${ }^{*}$ Speaker. 


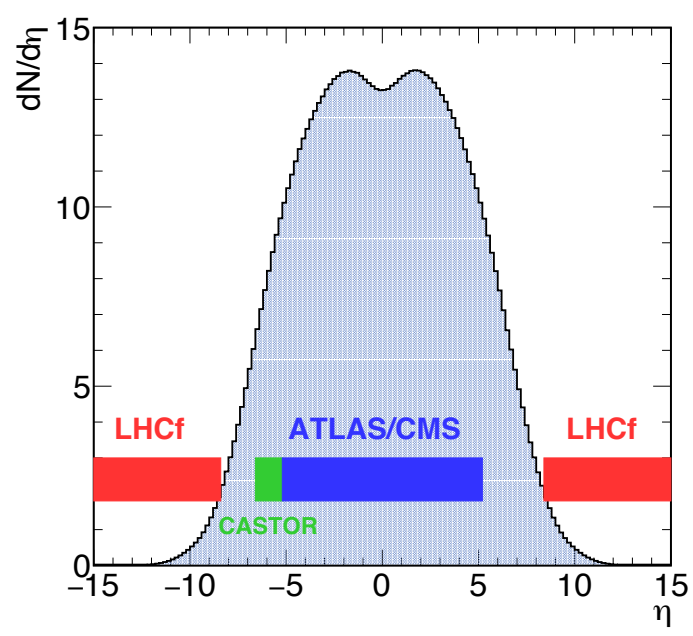

(a) Multiplicity

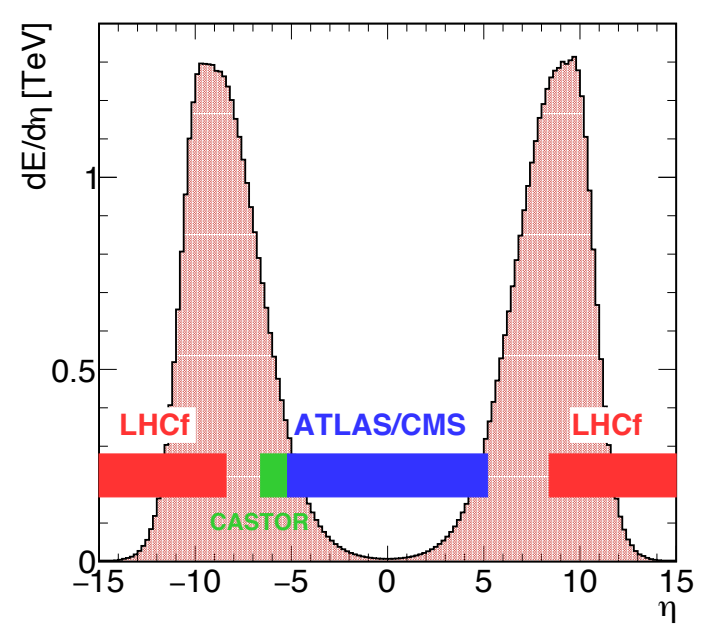

(b) Energy flow

Figure 1: Pseudorapidity dependencies of the multiplicity (left) and the energy flow (right) of all secondaries at $p p \sqrt{s}=13 \mathrm{TeV}$ collisions [?]. The color bands shows the acceptance of the experiments.

\section{Introduction}

A hadronic interaction model is one of the key tools for ground-based cosmic-ray experiments. High energy cosmic-rays arriving at the earth interact atmosphere and induce extensive air-showers. High energy cosmic-ray experiments observe air showers and reconstruct energies, arrival directions, and chemical compositions of primary cosmic-rays from the data measured by ground-based detectors such as a particle detector array and/or Cherenkov telescopes. Hadronic interaction models are used in Monte Carlo (MC) simulations for air shower developments. These models were tested and tuned with many results of collider experiments. However, inconsistencies between observed data and MC simulation were reported [1, 2].

Large Hadron Collider (LHC) is operating since 2009 to provide unique opportunities to measure the hadronic interactions at the center-of-mass collision energy $\sqrt{s}$ from $0.9 \mathrm{TeV}$ up to $14 \mathrm{TeV}$. The maximum collision energy of LHC corresponds to $10^{17} \mathrm{eV}$ in the laboratory system. The LHC forward (LHCf) experiment [3] was designed to measure the production of neutral particles in the very forward region of collisions at LHC. Most of energetic particles, which are important in air-shower development, produce in the very forward region as shown in Fig. 1. The LHCf provides critical data to test and tune hadronic interaction models.. The LHCf has obtained data with $p p$ collisions at several collision energies from $0.9 \mathrm{TeV}$ to $13 \mathrm{TeV}$ and with $p \mathrm{~Pb}$ collisions at $\sqrt{s_{N N}}=5 \mathrm{TeV}$ and $8 \mathrm{TeV}$. In this paper, recent results of the LHCf experiment with pp collisions at $\sqrt{s}=13 \mathrm{TeV}$ and future prospects are presented.

\section{The experimental setup}

The LHCf experiment has two independent detectors, so called Arm1 and Arm2. They were installed $\pm 140 \mathrm{~m}$ from the ATLAS interaction point. At that point, the beam pipes makes a transition from a big-diameter pipe to two small-diameter pipes which connect to the LHC arc. The 


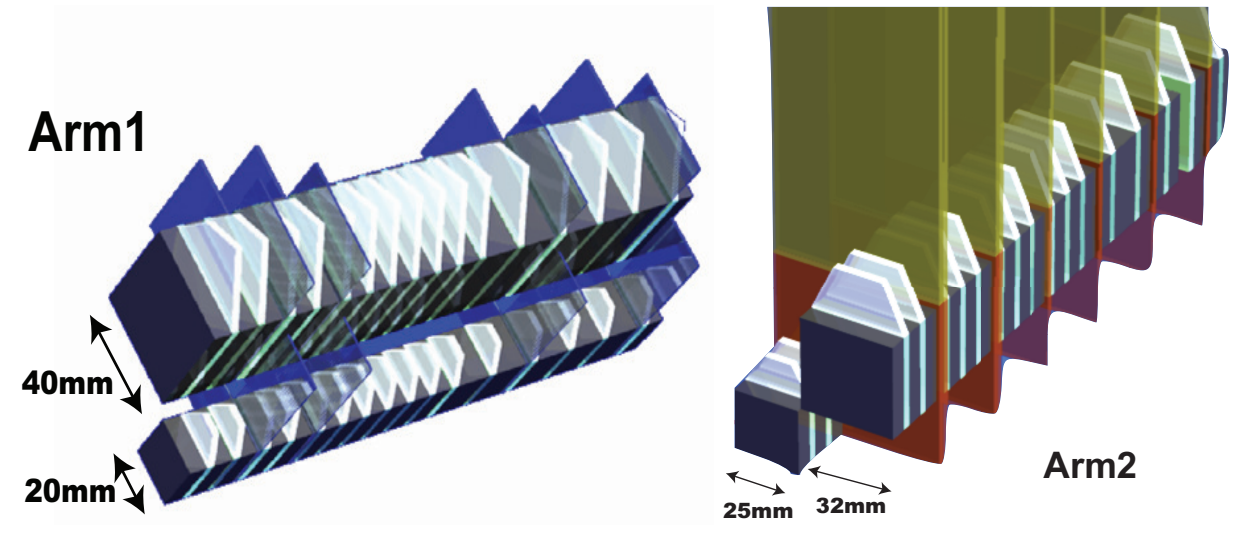

Figure 2: Schematic views of the Arm1 (left) and Arm2 (right) detectors

detectors were inserted into the $10 \mathrm{~cm}$ gap between the small-diameter pipes to view the zero degree of collisions. Because of the presence of dipole magnets located between IP1 and the detectors, the LHCf detectors were able to measure only neutral particles like photons and neutrons.

Each LHCf detector has two sampling and imaging calorimeter towers which are consisted of tungsten plates, 16 scintillator layers for shower sampling and four position sensitive layers for measurement of shower position [5]. The transverse sizes of the towers are $20 \mathrm{~mm} \times 20 \mathrm{~mm}$ and $40 \mathrm{~mm} \times 40 \mathrm{~mm}$ in Arm1 and $25 \mathrm{~mm} \times 25 \mathrm{~mm}$ and $32 \mathrm{~mm} \times 32 \mathrm{~mm}$ in Arm2. In nominal operations, the small-size calorimeters were positioned on the zero degree of collisions. The pseudorapidity coverage of the detectors is $\eta>8.4$. The energy resolution is better than $5 \%$ for photons and about $40 \%$ for neutrons. The position resolutions for photons and neutrons are better than $0.2 \mathrm{~mm}$ and $1 \mathrm{~mm}$, respectively.

The LHCf had an operation with $p p$ collisions at $\sqrt{s}=13 \mathrm{TeV}$ in June 2015. To avoiding the event pileup, the operation was performed with very low luminosity about $10^{29} \mathrm{~cm}^{2} \mathrm{~s}^{-1}$. After a 3 days operation, the detectors have been removed from the LHC tunnel. During the operation, the LHCf final trigger signals except pedestal and calibration triggers were sent to the ATLAS DAQ and fired ATLAS triggers.

\section{Resent results}

\subsection{Forward photon measurement at $p p, \sqrt{s}=13 \mathrm{TeV}$}

A part of the data, taken on June $12-13$, was analyzed to measure the inclusive forward photon energy spectra in $p p$ collisions at $\sqrt{s}=13 \mathrm{TeV}$. The details of the analysis and the result are found in Ref. [6]. In the analysis, photon events were selected by using a PID estimator, $L_{90 \%}$, which is defined as the longitudinal depth, in units of radiation length $\left(X_{0}\right)$, at which the integral of the energy deposition in a calorimeter reached $90 \%$ of the total. Figure 3 shows the $L_{90 \%}$ distribution for the events with the reconstructed energy between $1.1 \mathrm{TeV}$ and $1.2 \mathrm{TeV}$. The distribution was fitted with MC templates for pure photon events and pure neutron events to estimate the selection efficiency and purity of photons after applying the PID selection.

Figure 4 shows the measured inclusive photon energy spectra at the pseudo-rapidity regions $\eta>10.94$ and $8.81<\eta<8.99$. The results were taken from the combination of the Arm1 and 


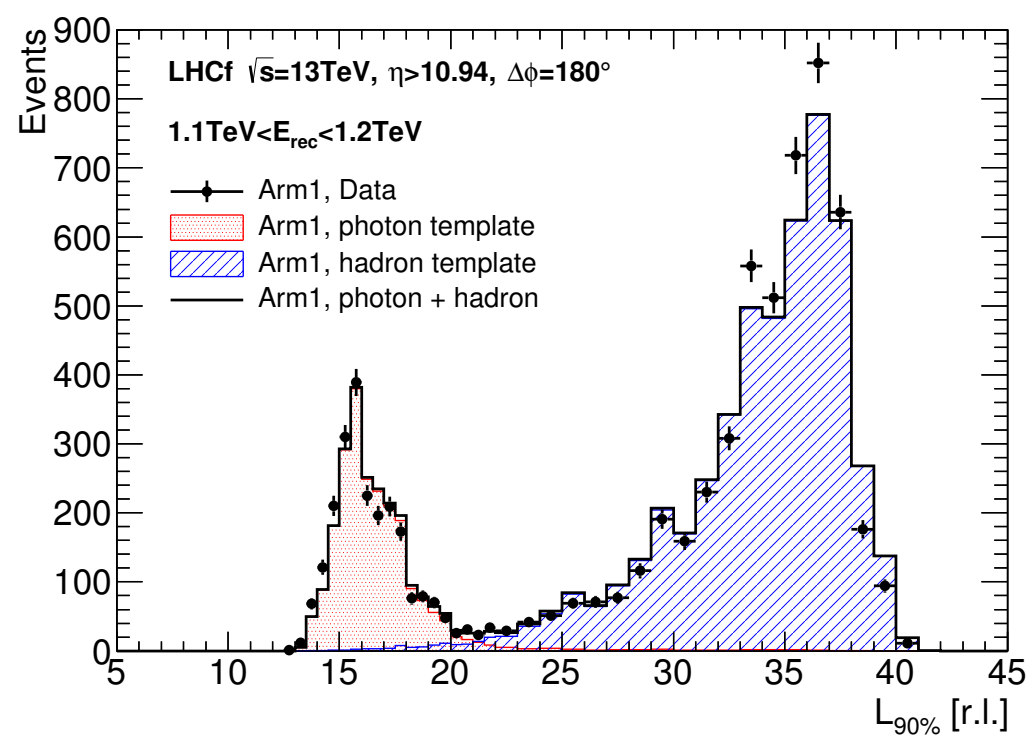

Figure 3: The $L_{90 \%}$ distribution in Arm 1 for the events with the reconstructed energy between $1.1 \mathrm{TeV}$ and 1.2 $\mathrm{TeV}$ [6]. The black points show the experimental data with the statistical error bars. The red and the blue colored lines correspond to the template distributions obtained from the MC simulation for photons and neutrons, respectively. The black line shows the total of the template distributions. These distributions were normalized by the results of the template fitting.

Arm2 results. The black points show the experimental results and the colored lines show the predictions of several hadronic interaction models, QGSJET II-04 [7], EPOS-LHC[8], DPMJET3.06[9], SIBYLL 2.3[10], and PYTHIA 8.212[11]. As a general conclusion, we found no model can reproduce our data perfectly. In the pseudorapidity region $\eta>10.94$ which covers the zero degree of collisions, the QGSJET II-04 and EPOS-LHC models show the best agreements overall with the data. PYTHIA 8.212 shows a good agreement with the data from the lowest energy bin to near the $3 \mathrm{TeV}$ bin although it clearly predicts higher flux than the data in the energy region greater than 3 $\mathrm{TeV}$. In the pseudorapidity region $8.81<\eta<8.99$ which corresponds to a off-center region, the EPOS-LHC and PYTHIA 8.212 models show good agreement with the data except at the highenergy end above $3 \mathrm{TeV}$. QGSJET-II-04 shows lower flux than the data. The trends of SIBYLL 2.3 from the data are different between the two rapidity regions. It is due to the SIBYLL's larger mean value of transverse momentum than both the data and the other models.

Energy flow of forward photons was calculated by integrating the energy spectra measured by the LHCf-Arm1 detector. The analysis was extended to other three pseudorapidity regions: 8.52 $<\eta<8.66,8.66<\eta<8.81$, and $8.99<\eta<9.22$. The result was compared with the model predictions as shown in Fig. 5. EPOS-LHC and SIBYLL 2.3 show good agreements with the data around the peak, while QGSJET-II-04 predicts lower energy flow than the data. These agreement of EPOS-LHC and SIBYLL 2.3 is related to the agreement of inclusive photon spectra with the data in the low energy region at $8.81<\eta<8.99$ as shown in Fig. 4.

\subsection{Forward neutron measurement at $p p, \sqrt{s}=13 \mathrm{TeV}$}

Forward neutron energy spectra at the pseudorapidity ranges, $\eta>10.84,8.99<\eta<9.22$, 

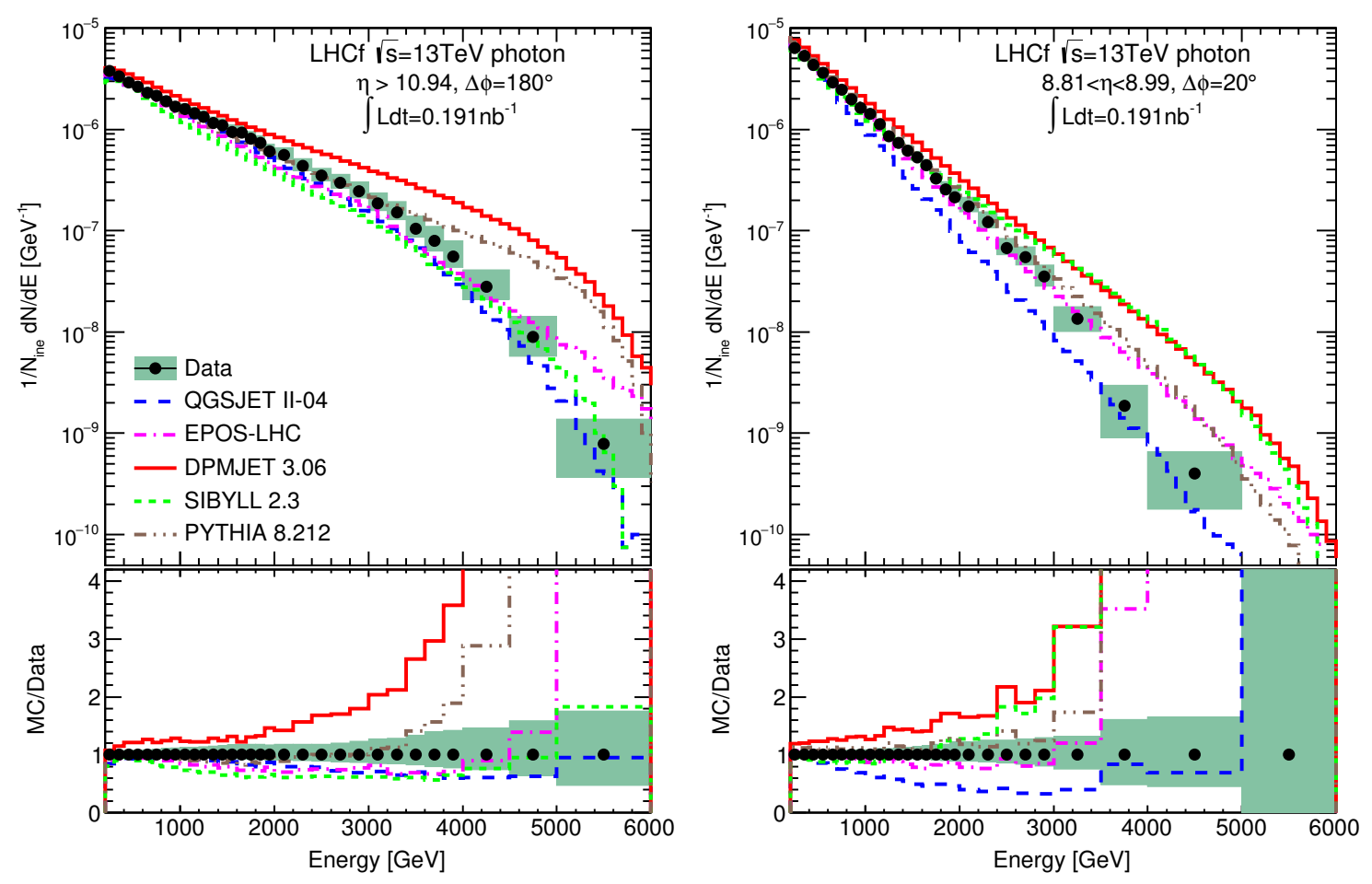

Figure 4: Comparison of the photon spectra obtained from the experimental data and MC predictions [6]. The top panels show the energy spectra and the bottom panels show the ratio of MC predictions to the data. The hatched areas indicate the total uncertainties of experimental data including the statistical and the systematic uncertainties.

and $8.81<\eta<8.99$, were measured by the LHCf-Arm2 detector [12]. For the PID selection in the neutron analysis, a slightly modified PID estimator $L_{2 D}$ was used, which was defined as $L_{2 D}=L_{90 \%}-0.25 L_{20 \%}$, where $L_{20 \%}$ is the calorimeter depth containing $20 \%$ of the total energy deposit measured by the calorimeter. Figure 6 show the experimental results. The obtained spectra were compared with the predictions of several interaction models. At $\eta>10.8$, only QGSJET-I I-04 and EPOS-LHC reproduce the tendency of increasing flux observed in the data in the high energy region, while the other models predict decreasing flux. At $\eta<9.22$ and $8.81<\eta<$ 8.99, QGSJET-II-04 and EPOS-LHC show good overall agreements with the experimental data. Especially, EPOS-LHC have a good agreement with the data in the energy region around $1.5 \mathrm{TeV}$. 


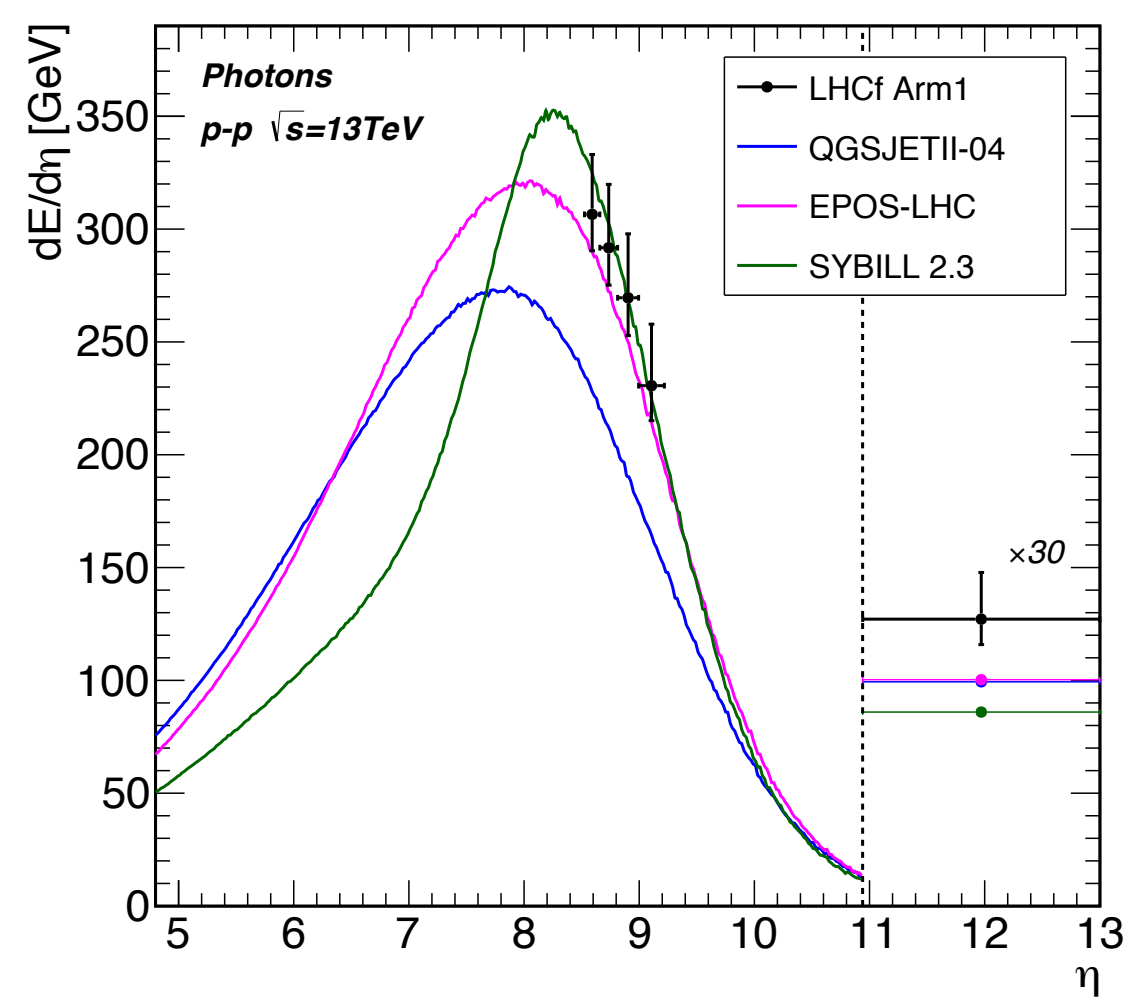

Figure 5: Pseudorapidity dependence of photon energy flow measured by the LHCf-Arm1 detector [4]. The experimental results are represented by the black points and the MC predictions of EPOS-LHC, QGSJET II-04 and SIBYLL 2.3 are shown as the colored lines. The point above $\eta=10.94$ is scaled by a factor of 30 for clarity and it is artificially limited to $\hat{I}^{\circ}=13$. 

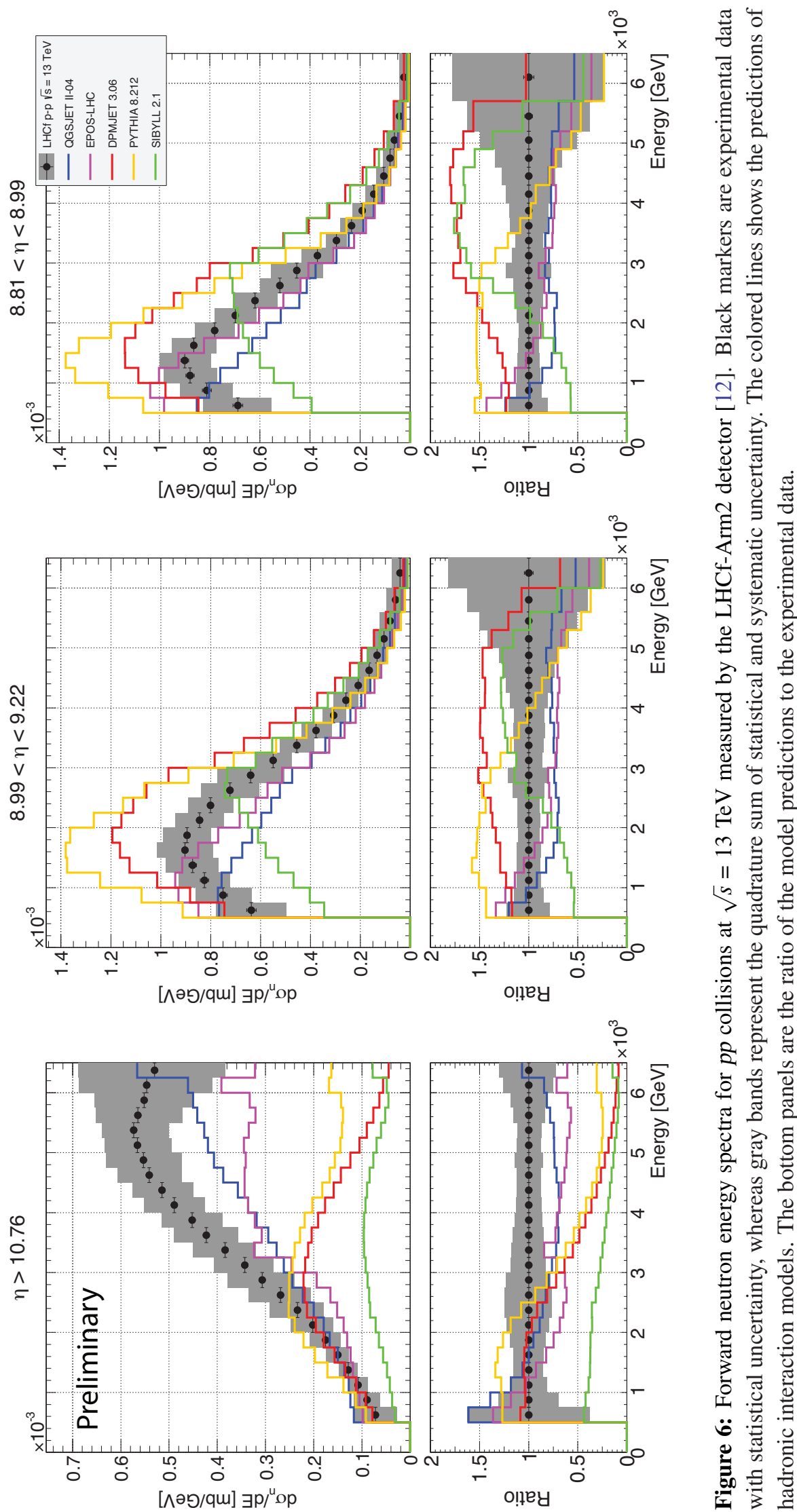


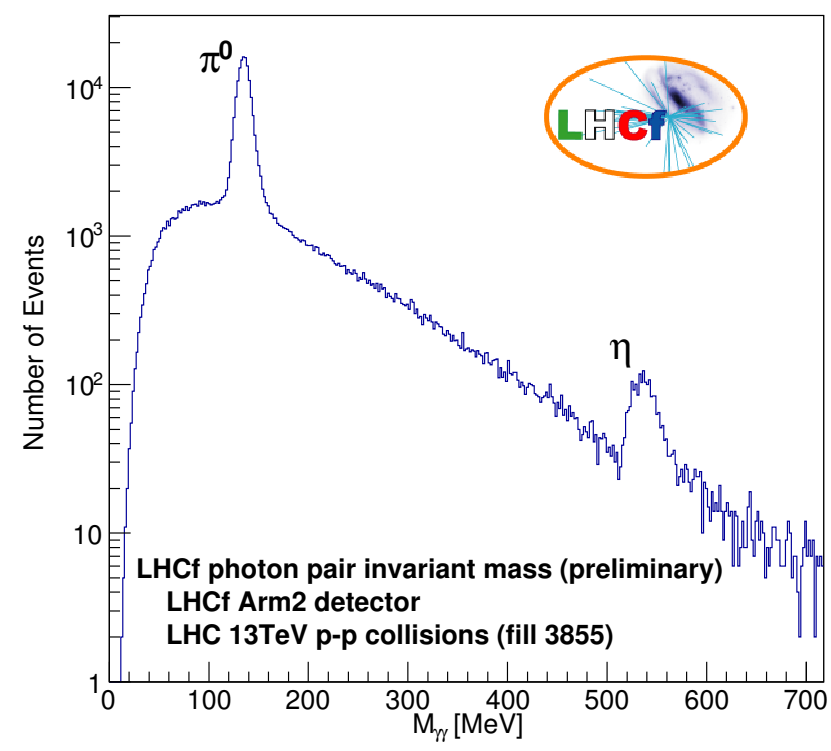

Figure 7: The invariant distribution of photon pair events measured with pp collisions at $\sqrt{s}=13 \mathrm{TeV}$. The two peaks on the distribution correspond to the mass of $\pi^{0}$ and $\eta$ mesons.

\section{Future prospects}

\subsection{On-going analyses}

The next target of the analysis after completion of the neutron analysis is the measurement of $\pi^{0}$ differential cross-section with the data at $p p, \sqrt{s}=13 \mathrm{TeV}$. Most of photons produced in hadronic interactions are from $\pi^{0}$ decays to photon pairs. The kinematics of a produced $\pi^{0}$ is reconstructed by measuring a photon pair from the $\pi^{0}$ decay by the two calorimeter towers (type- 1 event) or one calorimeter tower (type- 2 event). The minimum energy of detectable $\pi^{0}$ s in type 1 events is about $500 \mathrm{GeV}$, which is much lower than the minimum energy in type- 2 events, $2 \mathrm{TeV}$. Therefore, the type- 1 events are used for the measurement of low-energy $\pi^{0}$ 's and the type- $2 \pi^{0}$ events are used for high-energy $\pi^{0}$. The Feynman scaling hypothesis of forward $\pi^{0}$ production cross-section was tested with the data in $p p$ collisions at $\sqrt{s}=2.76 \mathrm{TeV}$ and $7 \mathrm{TeV}$ in Ref. [13]. It is needed to be test with the data at $\sqrt{s}=13 \mathrm{TeV}$ also.

Additionally, the analysis for the measurement of production cross section of forward $\eta$ mesons is on-going. The $\eta$ meson has the mass of $547 \mathrm{MeV}$, which is 4 times heavier than $\pi^{0}$. A $\eta$ decays to $\gamma \gamma(39.4 \%), 3 \pi^{0}(32.7 \%)$, or $\pi^{+} \pi^{-} \pi^{0}(22.9 \%)$. Therefore, $\eta$ mesons produced in interactions between cosmic-rays and atmosphere induce electromagnetic showers. The production cross section of $\eta$ is predicted to be about $10 \%$ of $\pi^{0}$ 's cross section by hadronic interaction models, however, the production cross section of very forward $\eta$ has been never measured with $p p$ colliders. The event reconstruction method for $\pi^{0}$ type- 1 events can be applied for $\eta$ measurement also. The minimum energy of detectable $\eta$ mesons is $2 \mathrm{TeV}$. Figure 7 shows the invariant mass distribution of type- 1 events. $\eta$ candidates events are selected with the reconstructed mass cut. 


\subsection{Joint analysis with the ATLAS experiment}

A joint analysis of both ATLAS and LHCf collaborations is on-going with the data set taken with $p p$ collisions at $\sqrt{s}=13 \mathrm{TeV}$. Combining the LHCf data with information of central activities of collisions provided by ATLAS, hadronic processes of forward particle production can be studied. The first target of the joint analysis is to measure the contribution of diffractive processes on the forward particle production. The uncertainty of diffractive processes implemented in the models is one of the possible sources of the discrepancies between the data and the model prediction on the photon and neutron results. Figure 8 shows the forward photon energy spectra predicted by several interaction models with the event categorization to total, non-diffraction, and diffraction by using the flag of event generator's outputs. The discrepancy of diffractive spectra between the models is large at $\eta>10.94$, whereas the discrepancy of total spectra between the models except PYTHIA 8212DL is relatively small. A Monte-Carlo (MC) simulation study [14] indicates a simple method to separate the contributions of diffractive and non-diffractive processes. By applying the event selection, no-charged particle production with $p_{T}>100 \mathrm{MeV}$ at $|\eta|<2.5$, non-diffractive events are rejected with keeping more than 50\% detection efficiency of diffractive events and more then $99 \%$ purity, which is defined as the fraction of the non-diffractive events to the selected events.

This event selection is realized by the data of the ATLAS inner tacker, which covers the pseudorapidity range $|\eta|<2.5$. The LHCf measured data are studied with categorization based on the number of tracks detected by ATLAS.

\subsection{Operation in 2016; $p$-Pb collisions at $\sqrt{s_{N N}}=8 \mathrm{TeV}$}

The LHCf experiment joined an LHC operation with $p$-Pb collisions in 2016. LHC provided $p \mathrm{~Pb}$ collisions with the collision energies per nucleon $\sqrt{s_{N N}}=5 \mathrm{TeV}$ and $8 \mathrm{TeV}$. The physics motivation of the operation is to measure the nuclear modification factors of forward particle production at the LHC-maximum collision energy. Thanks to factor 1.6 higher collision energy than $5 \mathrm{TeV}$, the LHCf detector covered a wider range of transverse momentum than the past $5 \mathrm{TeV}$ operation. The operation with $\sqrt{s_{N N}}=5 \mathrm{TeV}$ was performed also to confirm the results of the past operation [13] with the upgraded detector. It helps also to reduce systematic uncertainties related to the detector performance in analyses of comparison of $8 \mathrm{TeV}$ results with $5 \mathrm{TeV}$ results to test energy dependencies of forward particle production.

The Arm2 detector was installed to the LHC tunnel again in the beginning of November 2016. The detector was located on the p-remnant side of collisions. The operation has been successfully completed on November 25th. 25.3 M events and 16.7 M events were recoded with $p$-Pb collisions at $\sqrt{s_{N N}}=5 \mathrm{TeV}$ and $8 \mathrm{TeV}$, respectively. Figure 9 shows some results taken from the online analysis. The left figure shows the photon energy spectrum measured by the small calorimeter tower of the Arm2 detector. The right panel shows the hit map of the hadronic shower events. The beam center is located near the edge of the small calorimeter tower. During the operation, the common operation with the ATLAS experiment was performed. The event-by-event information of central activities measured by the ATLAS detector allow us to reject the contribution of ultra peripheral collisions to the forward particle production. 

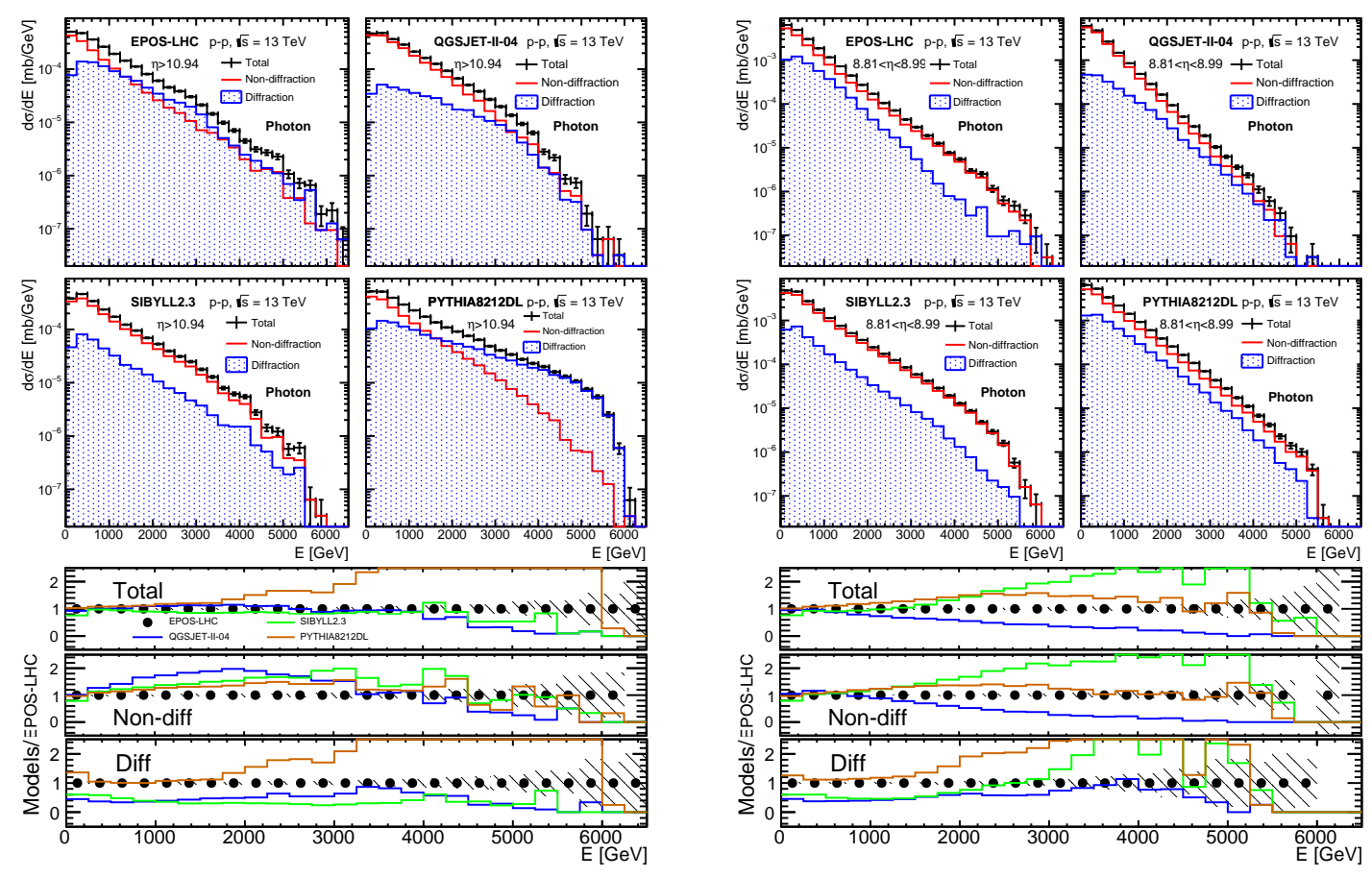

Figure 8: The total photon spectra (black) classified as non-diffracitve events (red) and diffractive events (blue) by using the flag of the event-generator outputs [14]. The bottom six plots show the ratios of the spectra of EPOS-LHC (black markers), QGSJET-II-04 (blue lines), SYBILL 2.3(green lines), and PYTHIA 8212DL (orange lines), to the spectrum of EPOS-LHC. The top, middle, and bottom plots correspond to total, non-diffractive, and diffractive events, respectively.

\subsection{The RHICf experimet; $p p$ collisions at $\sqrt{s}=510 \mathrm{GeV}$}

The RHIC forward (RHICf) experiment [15] measures the forward particles produced in $p p$ collisions at $\sqrt{s}=510 \mathrm{GeV}$ with the Relativistic Heavy Ion Collider (RHIC) located in Brookhaven National Laboratory (BNL), USA. The center-of-mass energy of $510 \mathrm{GeV}$ is equivalent to $10^{14} \mathrm{eV}$ in the laboratory system. Comparing with the results of the LHCf experiment, we can test the dependency of forward particle production on the collision energy and test hadronic interaction models with the data in the wide energy range from $10^{14} \mathrm{eV}$ to $10^{17} \mathrm{eV}$. It is important to extrapolate the LHCf-measured results from the LHC collision energy to the energy of UHECRs.

The RHICf collaboration was formed with most of the LHCf members and some members working at RHIC for the spin physics. For the RHICf operation, one of the LHCf detectors and the LHCf DAQ system were used. They were delivered from CERN to BNL after the LHCf operation in 2015. The detector was installed $18 \mathrm{~m}$ far from a RHIC interaction point where the STAR detector is located. Because of shorter distance between the detector and the interaction point than $140 \mathrm{~m}$ of the LHCf experiment, the RHICf detector is able to cover much wider $X_{F}-p_{T}$ phase space than the coverage of the LHCf operation at $p p, \sqrt{s}=900 \mathrm{GeV}$, which is similar as the coverage of the LHCf operation at $p p, \sqrt{s}=7 \mathrm{TeV}$. The RHICf took data successfully from September 23rd until September 26th, 2017. In the operation, more than 100 millions events were 

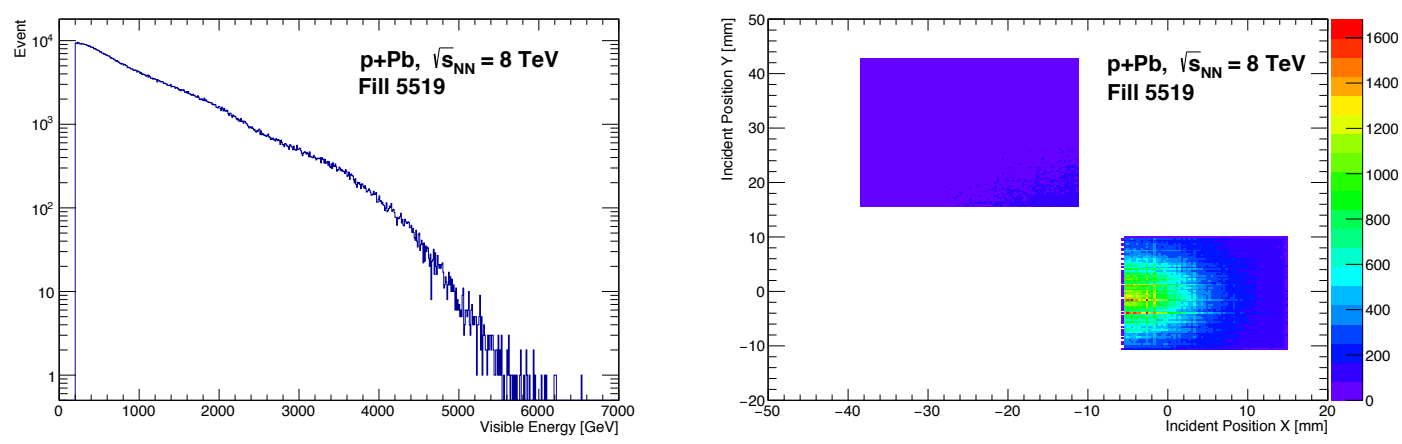

Figure 9: Results from the online analysis during the operation with $p$ - $\mathrm{Pb}$ collisions at $\sqrt{s_{N N}}=8 \mathrm{TeV}$. The left figure shows the photon energy spectrum measured by the small calorimeter tower of the Arm 2 detector. The right panel shows the hit map of the hadronic shower events.

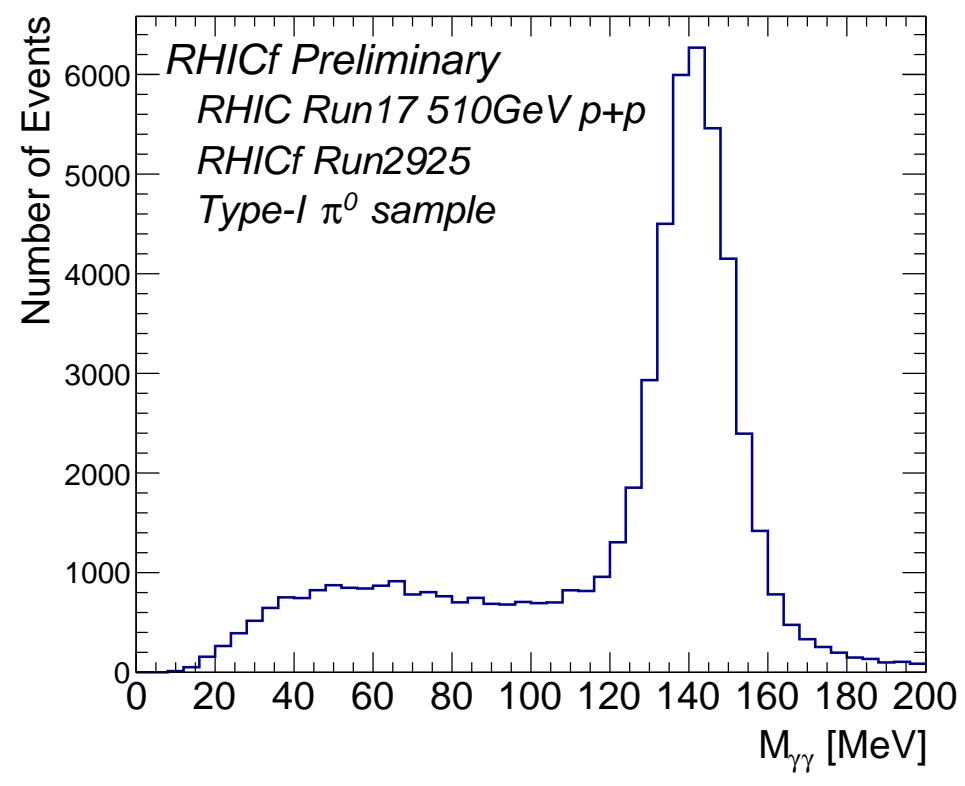

Figure 10: The invariant mass distribution of photon pair events measured in a RHICf run.

recoded with changing the detector position. Figure 10 shows a online analysis result, the invariant mass distribution reconstructed from photon pair events. The $\pi^{0}$ mass peak is clearly seen.

The common operation between the RHICf and STAR experiments was performed during the operation. The RHICf DAQ trigger signals were sent to the STAR DAQ system and their signals triggered the STAR DAQ. The common operation increases the physics potential of the RHICf experiment, for examples, diffractive study with the STAR central tracker and improvement of energy resolution for neutrons by combine-analysis with the STAR zero degree calorimeter.

\section{Summary}

The LHCf experiment had several operations with $p p$ and $p$-Pb collisions and tested hadronic 
interaction models used in MC simulation for cosmic-ray air showers. Post LHC models, QGSJET II04 and EPOS-LHC, show better agreement with the LHCf results for photons and neutrons at $p p$, $\sqrt{s}=13 \mathrm{TeV}$ than the other models. However, these model could not reproduce our data perfectly. Results from LHCf-ATLAS joint analyses and a measurement at RHIC will be provided near future. They are important to understand contribution of diffractive process to forward particle productions and the energy scaling of forward particle cross-section. LHCf keeps to provide critical data to test hadronic interaction models.

\section{Acknowledgments}

We thank the CERN staff and the ATLAS Collaboration for their essential contributions to the successful operation of LHCf. This work was partly supported by JSPS KAKENHI Grant Numbers JP26247037, JP23340076 and the joint research program of the Institute for Cosmic Ray Research (ICRR), University of Tokyo. This work was also supported by Istituto Nazionale di Fisica Nucleare (INFN) in Italy. Parts of this work were performed using the computer resource provided by ICRR (University of Tokyo), CERN and CNAF (INFN).

\section{References}

[1] Pierre Auger Collaboration, Muons in air showers at the Pierre Auger Observatory: Measurement of atmospheric production depth, Phys. Rev. D90 (2014), no. 1, 012012.

[2] Pierre Auger Collaboration, Depth of maximum of air-shower profiles at the Pierre Auger Observatory. I. Measurements at energies above $10^{17.8} \mathrm{eV}$, Phys. Rev. D90 (2014), no. 12, 122005.

[3] LHCf Collaboration, Technical design report of the LHCf experiment: Measurement of photons and neutral pions in the very forward region of LHC, (2006), CERN-LHCC-2006-004.

[4] Y. Makino, Measurement of the very-forward photon production in $13 \mathrm{TeV}$ proton-proton collisions at the LHC, PhD thesis, Nagoya University (2017), CERN-THESIS-2017-049.

[5] LHCf Collaboration, The LHCf detector at the CERN Large Hadron Collider, JINST 3 (2008), S08006

[6] LHCf Collaboration, Measurement of forward photon-energy spectra for ấĽ̌s $=13 \mathrm{TeV}$ proton-proton collisions with the LHCf detector, CERN-EP-2017-051, [hep-ex/170307678].

[7] Sergey Ostapchenko, Monte Carlo treatment of hadronic interactions in enhanced Pomeron scheme:I. QGSJET-II model, Phys. Rev. D83 (2011), 014018.

[8] T. Pierog, Iu. Karpenko, J. M. Katzy, E. Yatsenko, and K. Werner, EPOS LHC: Test of collective hadronization with data measured at the CERN Large Hadron Collider, Phys. Rev. C92 (2015), no. 3, 034906.

[9] Fritz W. Bopp, J. Ranft, R. Engel, and S. Roesler, Antiparticle to Particle Production Ratios in Hadron-Hadron and d-Au Collisions in the DPMJET-III Monte Carlo, Phys. Rev. C77 (2008), 014904.

[10] Eun-Joo Ahn, Ralph Engel, Thomas K. Gaisser, Paolo Lipari, and Todor Stanev, Cosmic ray interaction event generator SIBYLL 2.1, Phys. Rev. D80 (2009), 094003.

[11] Torbjorn Sjostrand, Stephen Mrenna, and Peter Z. Skands, A Brief Introduction to PYTHIA 8.1, Comput. Phys. Commun. 178 (2008), 852-867. 
[12] E. Berti, Measurement of energy spectra relative to neutrons produced at very small angle in $\sqrt{s}=13$ TeV proton-proton collisions using the LHCf Arm 2 detector, $\mathrm{PhD}$ thesis, University of Florence (2017) CERN-THESIS-2017-035.

[13] LHCf Collaboration, Measurements of longitudinal and transverse momentum distributions for neutral pions in the forward-rapidity region with the LHCf detector, Phys. Rev. D 94 (2016) 032007.

[14] Qi-Dong Zhou, Yoshitaka Itow, Hiroaki Menjo, and Takashi Sako, Monte Carlo study of particle production in diffractive proton-proton collisions at $\sqrt{s}=13 \mathrm{TeV}$ with the very forward detector combined with central information, Eur. Phys. J. C77 (2017), no. 4, 212.

[15] RHICf Collaboration, Measurement of very forward particle production at RHIC with $\sqrt{s}=510 \mathrm{GeV}$ proton-proton collisions, Proceedings of ICRC 2017. 